\title{
Nonlinear reflection from liquid crystal elastomers
}

\author{
Michele F. Moreira, Paul Luchette, and Peter Palffy-Muhoray ${ }^{*}$ \\ Liquid Crystal Institute, Kent State University, Kent, OH 44242, USA
}

Received September 30, 2010; accepted September 30, 2010; published September 30, 2010

\begin{abstract}
We have studied light induced deformations in dye doped liquid crystal elastomers. At high intensities, the incident light causes the elastomer to deform, and the reflected light from the deformed elastomer surface produces an intensity dependent diffraction pattern in the far field. We discuss the experimental observations, and relate these to the processes leading to photoinduced surface relief.
\end{abstract}

Liquid crystal elastomers (LCEs) are solid liquid crystals; they are cross-linked rubber networks with orientationally ordered constituents. Their salient feature is the coupling between orientational order and mechanical strain. This coupling provides a new way of changing orientational order and hence modifying the optical and physical properties of samples by mechanical strain. On the other hand, changing the orientational order via external fields can give rise to mechanical strain and changes in the sample shape. This can result in intensity dependent reflection and diffraction, similar to selffocusing/defocusing in transmissive nonolinear materials. This effect may be useful for adaptive optics and nonlinear optical devices.

The nematic LCE samples were prepared using the familiar two stage preparation technique developed by Finkelmann [1]. Briefly, the liquid crystal monomer, cross-linker, siloxane polymer backbone, disperse orange 3 (4-(4-nitrophenylazo)aniline) dye (DO3 from Aldrich), and platinum catalyst were dissolved in toluene and were spun in a custom spin casting apparatus for 1 hour at $65^{\circ} \mathrm{C}$, forming a partially reacted swollen film. Figure 1 shows the chemical structures of the constituents of the nematic LCE and of the dye. The dye was used without any further purification; the DO3 molecule is known to align with the director in nematic liquid crystals. The film was removed from the spin caster, cut into sections approximately $4 \mathrm{~cm}$ long, and the individual sections were suspended from a rigid support. To obtain a monodomain film, the LCE network was aligned by stretching, using weights of approximately $0.25 \mathrm{~g}$ attached to one end of the film. Weight was incrementally added over the course of 6 hours, up to a final value of approximately $1.5 \mathrm{~g}$. In order to obtain polydomain samples, the film was suspended without stretching for

\footnotetext{
*E-mail: mpalffy@cpip.kent.edu
}

the same period of time. Both types of films were then cured in an oven for 72 hours at $40^{\circ} \mathrm{C}$. The resulting LCE films were doped with $0.2 \%$ DO3 with a cross-link density of $9 \%$. The nematic-isotropic transition temperature for mono- and polydomain films is $45^{\circ} \mathrm{C}$ and $50^{\circ} \mathrm{C}$, respectively. The monodomain samples consisted of a single well-aligned nematic domain; in polydomain samples, domain size is estimated to be $2 \mu \mathrm{m}$ at room temperature [2-3].

(a)

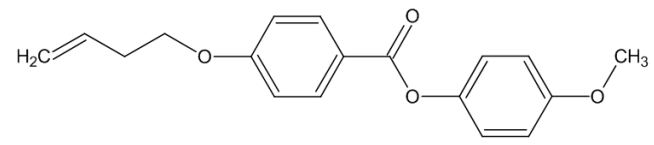

(b)

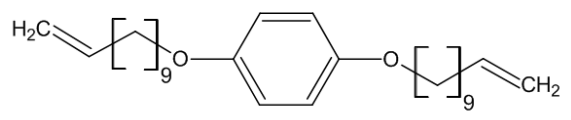

(c)<smiles>C[SiH2]O[Si](C)(C)O[SiH3]C</smiles>

(d)

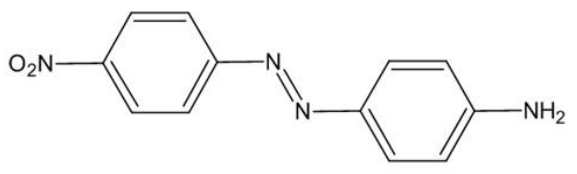

Fig. 1. Molecular structure of the (a) monomer, (b) cross-linker, (c) siloxane polymer, and (d) DO3 dye.

The experimental apparatus is shown in Fig. 2. The setup consisted of a CW Ar+ laser (Innova 100, Coherent) lasing at $\lambda=514 \mathrm{~nm}$ as the illumination source. A variable attenuator was placed in the beam to control the intensity of light incident on the sample. The incident beam was split by an unpolarized $5 \mathrm{~mm}$ diameter beam splitter. The intensity of beam reflected from the calibrated beam splitter was measured by an optical power meter (model $1830 \mathrm{C}$, Newport) to provide the laser power incident on 
the sample. The elastomer samples were placed on a microscope slide which had been painted black to eliminate back reflection from the glass. A lens with $2 \mathrm{~mm}$ focal length was used to focus the laser beam on the sample. The beam waist at the sample is approximately 6 $\mu \mathrm{m}$. The diffraction pattern was projected on a screen in the far field; image acquisition was via a CCD camera (Sumix SMX-M7x). The exposure time of the camera had to be increased for images pumped at higher power due to the low intensity of the diffraction pattern. The screen was located $70 \mathrm{~cm}$ from the elastomer sample.

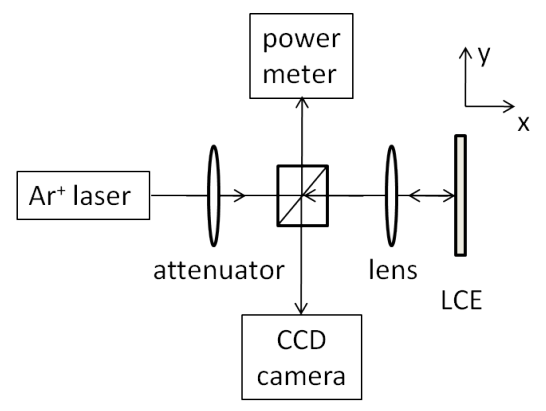

Fig. 2. Experimental setup.

The experimental results are shown in Fig. 3 and Fig. 4. Fig. 3 shows the images of the diffraction patterns obtained using monodomain LCE film for different pumping power $(10.7 \mathrm{~mW}, 14.6 \mathrm{~mW}, 19.7 \mathrm{~mW}$, and $24.3 \mathrm{~mW}$ ). The two dots observed on the right side of each images are spurious, due to reflection caused by the beam splitter. Fig. 4 shows the diffraction pattern obtained from the polydomain side-chain LCE for $11.7 \mathrm{~mW}, 19.3 \mathrm{~mW}, 22.1 \mathrm{~mW}$ and $26 \mathrm{~mW}$.

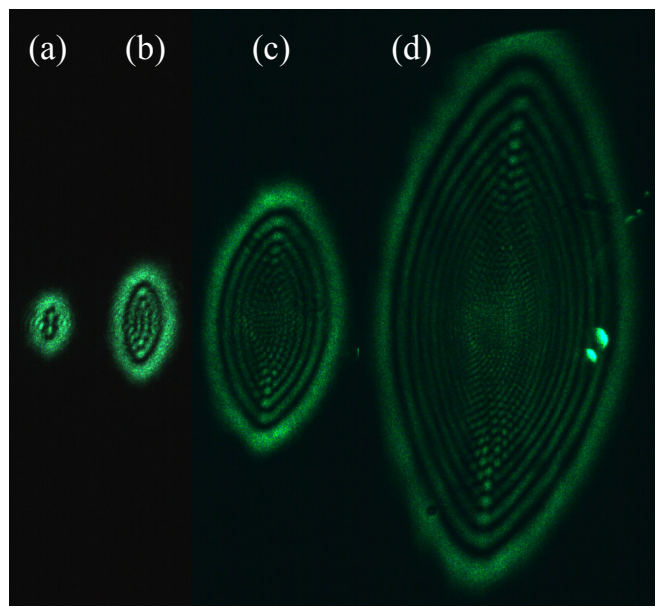

Fig. 3. Diffraction patterns from the monodomain film. The images correspond to the following pump powers: (a) $10.7 \mathrm{~mW}$, (b) $14.6 \mathrm{~mW}$, (c) $19.7 \mathrm{~mW}$, and (d) $24.3 \mathrm{~mW}$.

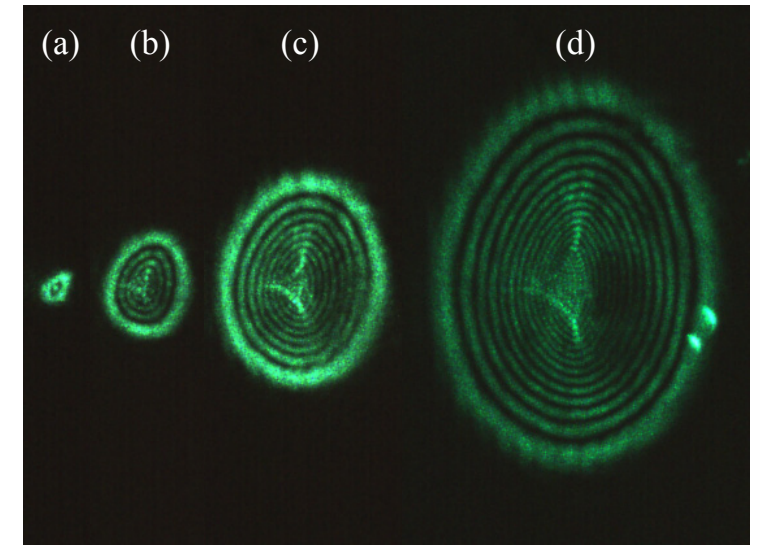

Fig. 4. Diffraction patterns from the polydomain film. The images correspond to the following pump powers: (a) $11.7 \mathrm{~mW}$, (b) $19.3 \mathrm{~mW}$, (c) $22.1 \mathrm{~mW}$, and (d) $26 \mathrm{~mW}$.

At very low intensities, the diffraction patterns are very similar for both mono- and polydomain films (these images are not shown). At approximately $11 \mathrm{~mW}$, the appearance of the diffraction patterns starts to differ (Figs. 3a and 4a). The monodomain diffraction pattern begins to show a vertical line at its center, in the direction parallel to the nematic director. At the same pump intensity, the polydomain film presents nearly circular rings. At higher intensities, the monodomain diffraction pattern shows a strongly elongated shape, with the direction of elongation parallel to the nematic director (Figs. 3b-d). The polydomain diffraction pattern shows nearly circular concentric rings, with what appear to be caustics near the center.

A wide variety of photomechanical effects have been observed in LCEs, ranging from samples swimming into the dark [4] and photoinduced oscillations [5] to lightdriven motors [6]. Here, the emphasis is to gain fundamental understanding of the photoinduced deformation process. Although intense laser light is expected to change the optical properties of bulk LCEs, the dominant effect here is assumed to be reflection from the photoinduced surface relief [7]. It is expected that surface relief is produced by the light induced change in the order parameter. If the degree of order is reduced, in samples such as ours, with oblate chain conformation, stresses appear which tend to cause contraction along the nematic director, and expansion in the perpendicular directions. To a good approximation, the volume is conserved. This stress field is relieved by the surface relief. Two mechanisms are expected to contribute to photoinduced changes in the order parameter. The first is laser heating due to linear absorption; as the temperature is increased, the degree of orientational order is reduced 
in thermotropic nematics. The second is trans-cis photoisomerization of the DO3 dye. Whereas the in the elongated trans-configuration the DO3 molecule resembles a nematic mesogen, and is expected to align with the other mesogens and the director, in the more compact cis-configuation, it functions more like an impurity, disrupting the order and reducing the nematicisotropic transition temperature. Both processes result in essentially the same phenomenon: photoinduced reduction of the degree of orientational order and subsequent contraction along the director and elongation in the perpendicular direction.

Numerical analysis is under way to determine the surface profile. This is made challenging by the loss of phase information in the intensity field of the diffraction patterns. Modelling the light induced surface deformations has been carried out (see Ref. [7]). Comparison of the experimentally determined surface profile with theoretical predictions will provide information about the goodness of the model description. The experimental results are also expected to provide useful information towards the design of devices utilizing photoinduced surface relief.
Nematic LCEs are strongly nonlinear, and show interesting intensity dependent reflection. They may be useful for applications such as adaptive optics and power limiting, as well as other applications requiring photoactuation.

This work was supported by the NSF under grant DMR 0606357.

\section{References}

[1] J. Kupfer, and H. Finkelmann, Makromol. Chem. Rapid Commun. 12 (12), 717-726 (1991).

[2] S.M. Clarke, E. Nishikawa, H. Finkelmann, and E.M. Terentjev, Macromol. Chem. Phys. 198, 3485-3498 (1997).

[3] S.M. Clarke, H. Finkelmann, and E.M. Terentjev, Macromolecules 31, 4862-4872 (1998).

[4] M. Camacho-Lopez, H. Finkelmann, P. Palffy-Muhoray, and M. Shelley, Nat. Mater. 3, 307-310 (2004).

[5] T.J. White et al., Soft Matter 4, 1796-1798 (2008).

[6] M. Yamada, et al., Angew. Chem., Int. Ed. 47, 4986-4988 (2008).

[7] M. Warner, and L. Mahadevan, Phys. Rev. Lett. 92, 134302 (2004). 